\title{
Superficially Spreading Endocervical Adenocarcinoma in situ with Multifocal Microscopic Involvement of the Endometrial Surface: A Case Report with Emphasis on the Potential for Misdiagnosis Based on Endometrial Curettage Specimens
}

Inwoo Hwang Jiyeon Lee Kyue-Hee Choi Jiheun Han Hyun-Soo Kim

Department of Pathology and Translational Genomics, Samsung Medical Center,

Sungkyunkwan University School of Medicine, Seoul, Republic of Korea

Keywords

Cervix · Endocervical adenocarcinoma in situ · Endometrium · Endometrioid carcinoma ·

Curettage

\begin{abstract}
Misdiagnosis of endocervical adenocarcinoma (EAC) as endometrial endometrioid carcinoma $(E E C)$ is one of the major concerns when evaluating endometrial curettage specimens. It is difficult to differentiate EAC involving the endometrium from EEC, particularly when the specimens have only a few small tumor fragments. We report a case of endocervical adenocarcinoma in situ (AIS) with multifocal microscopic involvement of the endometrium. The endometrial curettage specimen obtained from an 82-year-old woman consisted of a large volume of blood and fibrin, with small endometrial tissue fragments showing microscopic foci of atypical glandular proliferation. Based on the presence of complex glands with stratified mucin-poor columnar epithelium and intermediate-grade nuclear atypia, a preoperative diagnosis of grade $1 \mathrm{EEC}$ was made. However, the hysterectomy specimen revealed an endocervical AIS involving the endocervix and low uterine segment. Frequent mitotic figures and apoptotic bodies, characteristic of AIS, were present. The endometrium showed a few microscopic foci of atypical glandular proliferation involving the surface only. Their histological features were similar to those of the endocervical AIS. Immunohistochemically, the atypical glands exhibited block p16 positivity. The final diagnosis was a superficially spreading endocervical AIS with multifocal microscopic involvement of the endometrial surface epithelium. In summary, small tumor tissues in an endometrial curettage may lead to misdiagnosis of AIS or EAC as
\end{abstract}


EEC, especially when the pathologists are unaware of the possibility of microscopic endometrial involvement of AIS or EAC. The origin of the tumor can be correctly determined based on a combination of histological features and immunostaining. Endocervical AIS involving the endometrium should be included in the differential diagnosis of neoplastic glandular lesions in endometrial curettage specimens. An accurate diagnosis in these cases is important because of its significant implications for clinical management.

(C) 2020 The Author(s).

Published by S. Karger AG, Basel

\section{Introduction}

The endometrial versus endocervical origin of small tumor fragments in the endometrial curettage is difficult to distinguish by their morphological features alone [1]. Most frequently, the tumor shows atypical glandular proliferation of stratified columnar mucin-poor epithelium with intermediate-grade nuclear atypia, making it difficult to differentiate between endometrial endometrioid carcinoma (EEC) and endocervical adenocarcinoma (EAC). In particular, specimens with large amounts of blood, and a few small tumor fragments present a diagnostic challenge.

Endocervical adenocarcinoma in situ (AIS) is a premalignant lesion that can lead to the usual-type EAC [2].By definition, endocervical AIS normally involves only columnar epithelium and does not invade the cervical stroma [2,3]. It exhibits a variable anatomical distribution, extending along the endocervical canal for several centimeters, sometimes involving the entire circumference of the cervix. Nevertheless, an extension of the endocervical AIS proximal to the internal orifice is uncommon and the involvement of the endometrium, fallopian tube, or ovary is even rarer. However, several studies have reported a subset of endocervical tumors with minimal to no evidence of stromal invasion manifesting as metastatic tumors of the upper female genital tract [4-6].

In this study, we describe the detailed histological features and immunophenotype of an endocervical AIS involving the endometrial surface and initially misdiagnosed as EEC on the endometrial curettage specimen. A comprehensive analysis of the multifocal endometrial involvement of endocervical AIS will serve to improve the understanding of this rare condition and help pathologists make a correct diagnosis.

\section{Case Presentation}

An 82-year-old woman presented with vaginal bleeding. She had no previous history of gynecological disease. Abdominopelvic magnetic resonance imaging and transvaginal ultrasonography revealed hematometra without a definite mass. The bilateral adnexa were atrophied. No lymph node enlargement, peritoneal seeding, or abdominal metastasis was identified. Diagnostic endometrial curettage was performed. Histologically, the curetted specimen showed a large amount of blood and fibrin, consistent with hematometra, as seen with imaging (Fig. 1A-B). Several small endometrial tissue fragments comprised approximately $10 \%$ of the total specimen volume. A few foci of atypical glandular proliferation showed complex architectural pattern and intermediate-grade nuclear atypia (Fig. 1C), including mild enlargement, moderate pleomorphism, and inconspicuous nucleoli (Fig. 1D). The cytoplasm was scant. Several endometrial strips were also observed (Fig. 1E), and they showed the same degree of nuclear atypia as seen in the atypical glands attached to the fibrotic stroma (Fig. 1F). There was a transition from normal endometrial glandular epithelium to stratified columnar epithelium of variable thickness resembling normal proliferative endometrial glands. Even 


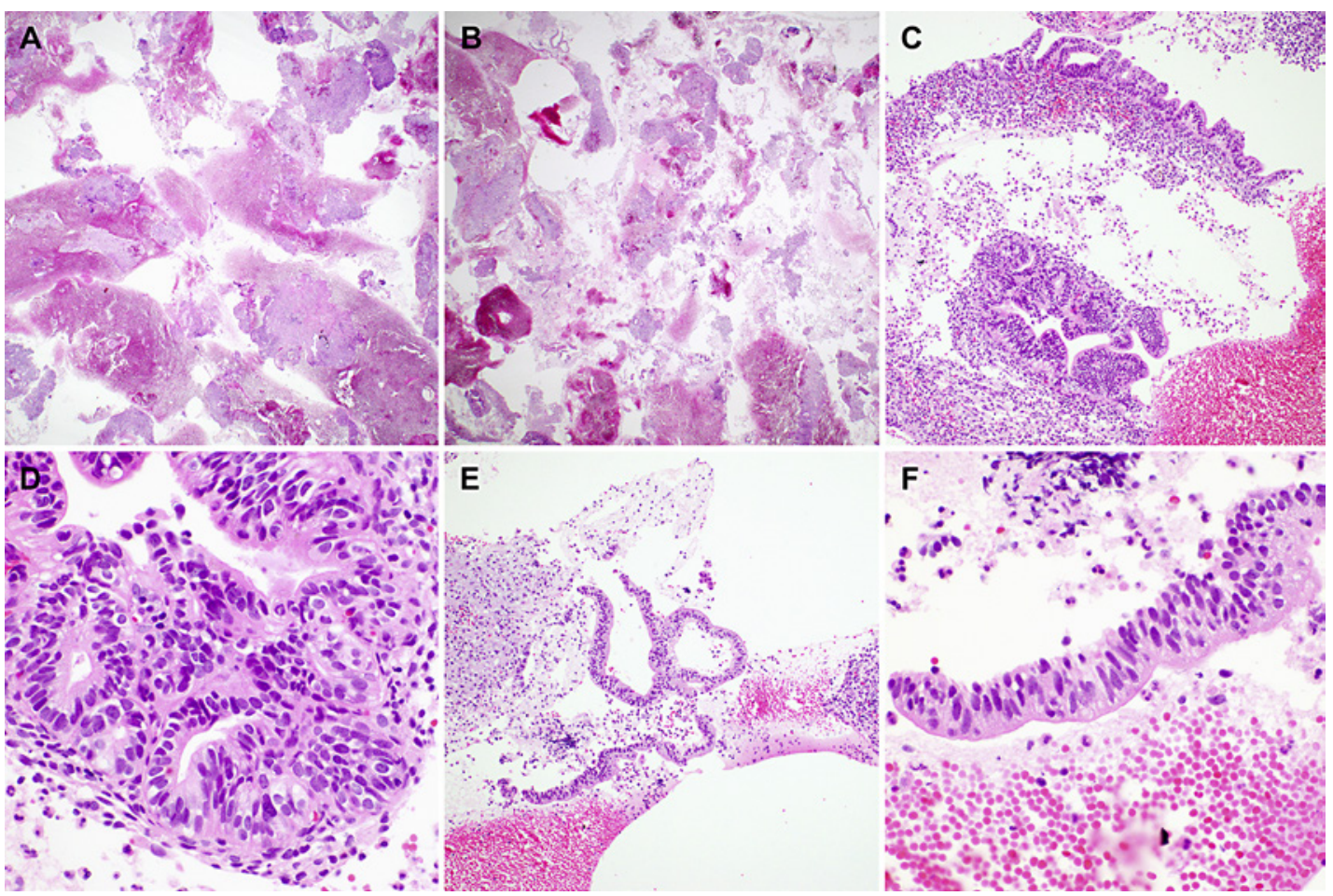

Fig. 1. Histological features of the endometrial curettage specimen. A-B Scanning magnification shows that the specimen is mainly composed of blood and fibrin. Small fragments of endometrial tissue are randomly scattered. C A few endometrial tissue fragments show complex glandular proliferation. D The atypical glands exhibit neoplastic epithelial proliferation characterized by nuclear stratification and loss of polarity. The nuclei show mild enlargement, moderate pleomorphism, and inconspicuous nucleoli. High-grade nuclear atypia or solid architecture is not seen. Based on these findings, the diagnosis of grade 1 endometrioid carcinoma of the endometrium is made. E Some fragmented endometrial strips also exhibit nuclear stratification. F On high-power magnification of image $\mathbf{E}$, the nuclear morphology and the degree of atypia observed in the strips are identical to those seen in the atypical glands in image $\mathbf{D}$. Note the ovoid-to-elongated nuclei with mildto-moderate pleomorphism and loss of polarity. Staining method: A-F, hematoxylin and eosin staining. Magnification: A-B, $\times 10$; C, $\times 50 ; \mathbf{D}, \times 400 ; \mathbf{E}, \times 40$; and $\mathbf{F}, \times 400$.

though the specimen appeared to consist mainly of blood at low-power magnification, a scant amount of atypical glands was observed at medium-to-high-power magnification. The diagnosis of grade 1 EEC was made. For the biopsy-proven but radiologically invisible endometrial carcinoma, the patient underwent total hysterectomy with bilateral salpingo-oophorectomy.

Grossly, no visible mass was identified in the endocervix and endometrial mucosa. The uterine serosa, parametrium, and bilateral adnexa were unremarkable. Histologically, the endocervix showed an AIS measuring $16 \mathrm{~mm}$. On low-power magnification, AIS spread horizontally up to the low uterine segment (Fig. 2A-B). No stromal invasion was identified. Frequent mitotic figures and apoptotic bodies, characteristic of human papillomavirus (HPV)related EAC, were present (Fig. 2C). The endometrium of the body and fundus showed a few separate microscopic foci of atypical glandular proliferation measuring $<2 \mathrm{~mm}$ and involving the endometrial surface (Fig. 2D). Based on the nuclear stratification, intraluminal papillary projection, and abundant eosinophilic cytoplasm, the atypical glandular epithelium was clearly demarcated from the adjacent inactive endometrial surface epithelium (Fig. 2E). No 


\section{Case Reports in Oncology}
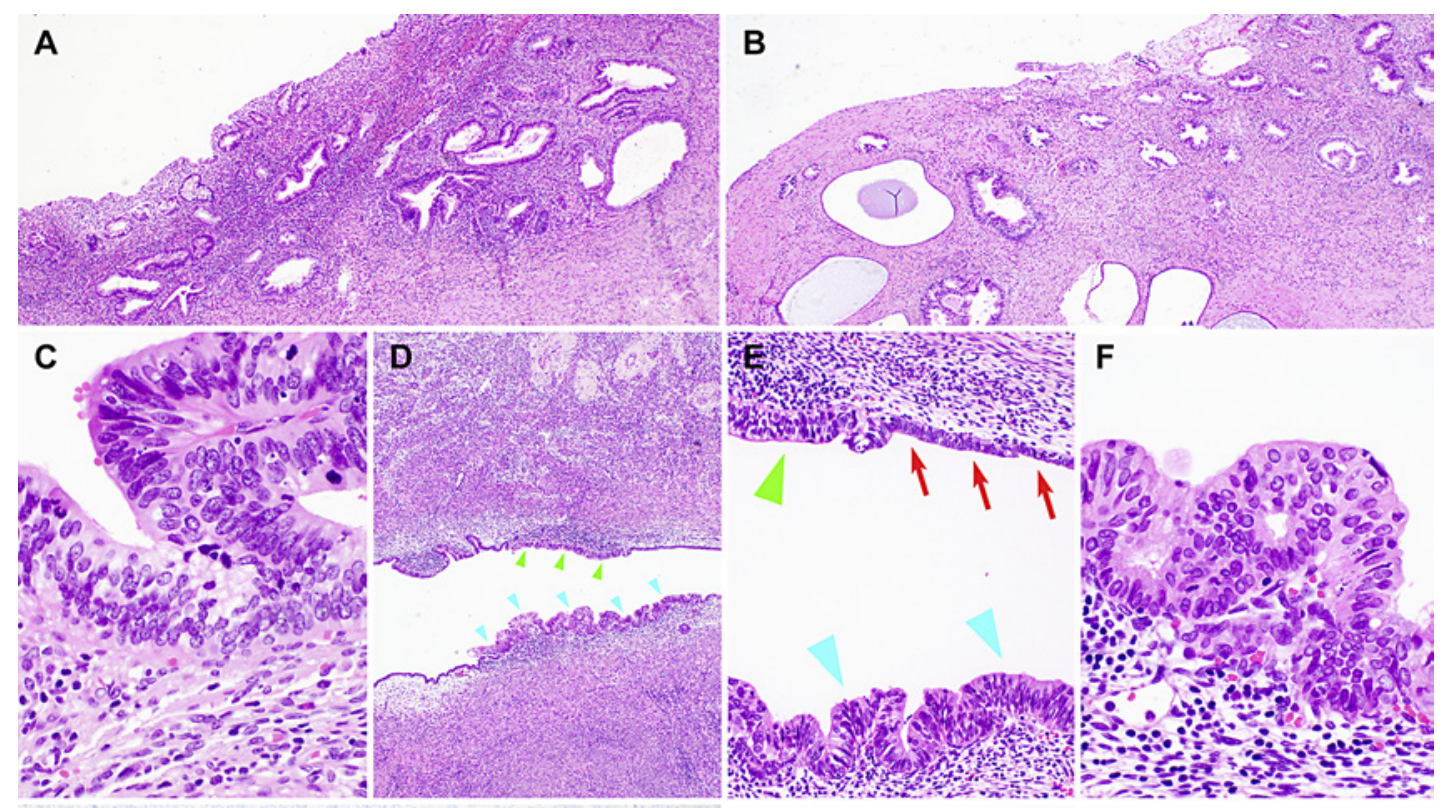

G

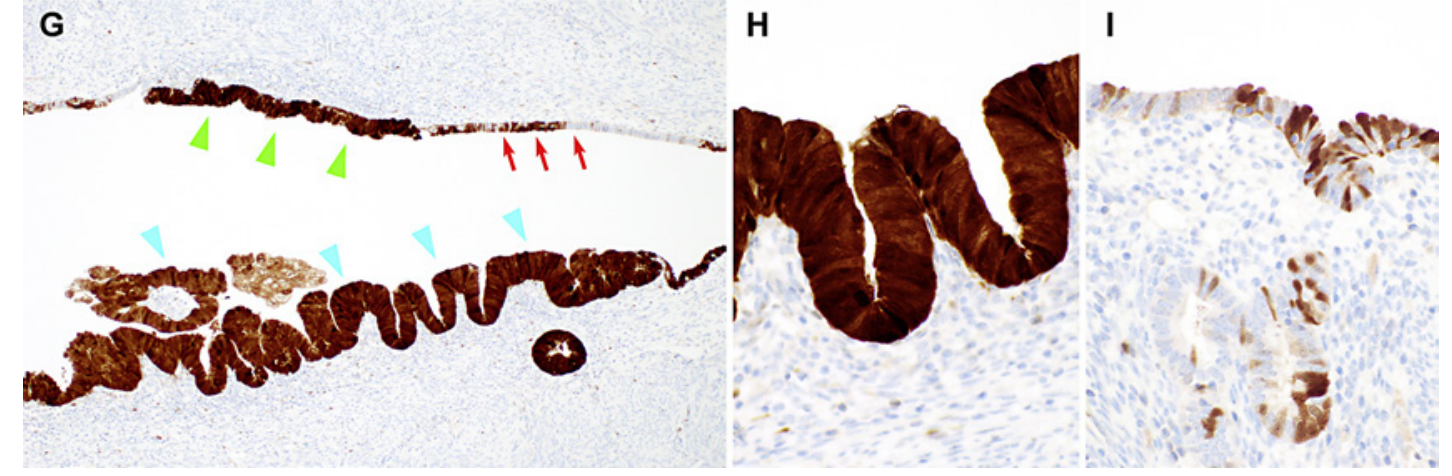

(c) 2020 The Author(s). Published by S. Karger AG, Basel www.karger.com/cro

Fig. 2. Histological features and immunostaining of the hysterectomy specimen. A-B The endocervix and low uterine segment show adenocarcinoma in situ (AIS) that had spread horizontally along the endocervical glands. C High-power magnification shows that the AIS has a stratified mucin-poor glandular epithelium with frequent mitotic figures and apoptotic bodies, both of which are characteristic features of human papillomavirus-associated tumors. D The endometrium displays microscopic $(<2 \mathrm{~mm})$ foci of atypical glandular proliferation (green and blue arrowheads) involving the surface epithelium only. E On medium-power magnification, this atypical epithelium (green and blue arrowheads) shows nuclear stratification and intraluminal papillary projection. They appear clearly demarcated from the adjacent endometrial glands (red arrows), consisting of a single layer of inactive, cuboidal to-low columnar epithelium. $\mathbf{F}$ The nuclear morphology and the degree of atypia in the atypical epithelium are comparable to those seen in endocervical AIS (image $\mathbf{C}$ ). G p16 immunostaining highlights the atypical areas with uniform and strong p16 expression in the nuclei (block positivity; green and blue arrowheads). In contrast, the adjacent inactive endometrial surface epithelium exhibits patchy p16 positivity (red arrows). H-I High-power magnification shows block p16 positivity in the AIS (H), and patchy p16 positivity in the uninvolved epithelium (I). Staining method: A-F, hematoxylin and eosin staining; G-I, polymer method (immunostaining). Magnification: A-B, $\times 40 ; \mathbf{C}, \times 400 ; \mathbf{D}, \times 40 ; \mathbf{E}$, $\times 100 ; \mathbf{F}, \times 400 ; \mathbf{G}, \times 100 ;$ and $\mathbf{H}-\mathrm{I}, \times 400$.

stromal invasion was noted. On high-power magnification, the nuclear morphology and the degree of atypia were similar to those in endocervical AIS (Fig. 2F). Mitotic figures and apoptotic bodies were detected, although not as frequent as observed in the endocervical AIS. Immunostaining revealed block 16 positivity in the atypical gland on the endometrial surface 


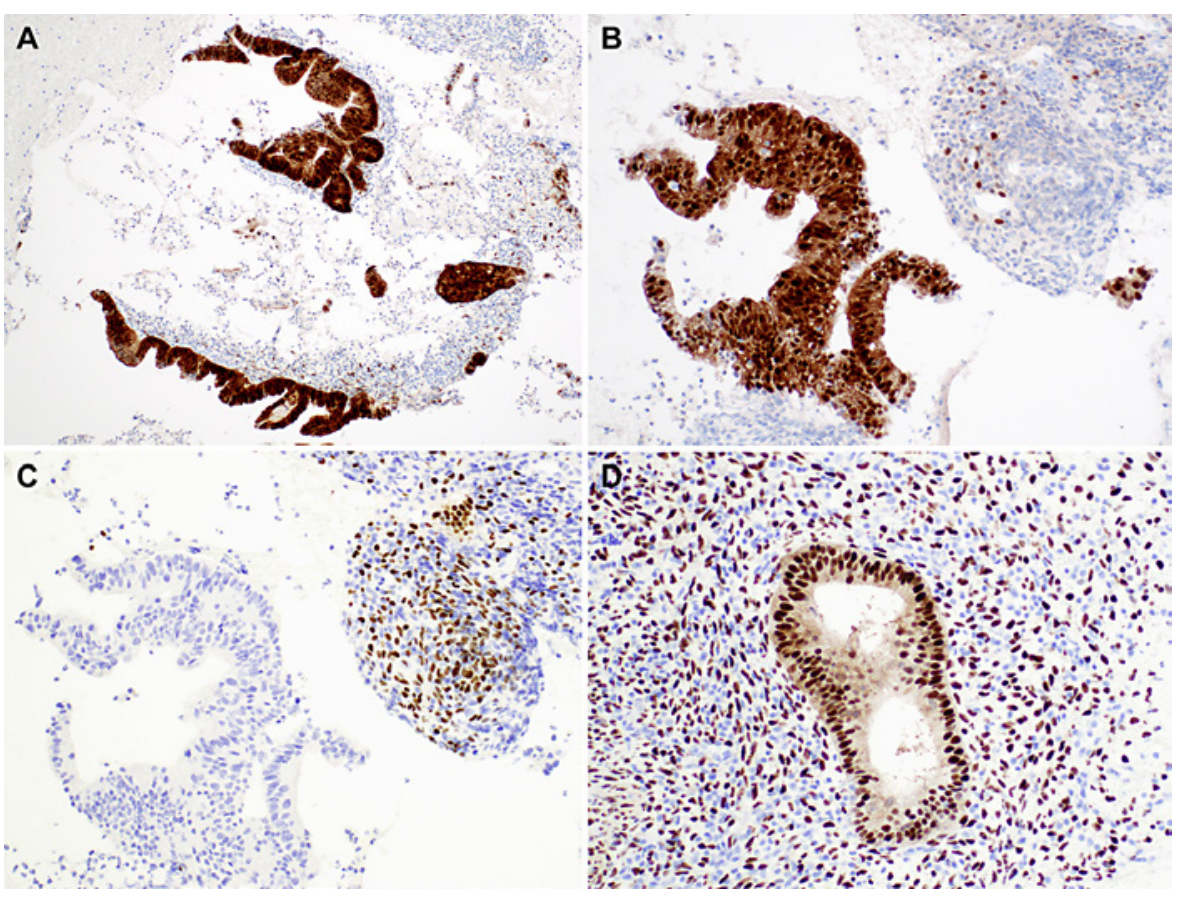

Fig. 3. Immunostaining of the endometrial curettage specimen. A Block p16 positivity confirms the diagnosis of adenocarcinoma in situ (AIS) of endocervical origin. B The atypical glands initially misdiagnosed as endometrial endometrioid carcinoma (left half) also show a uniform and strong expression of p16 in their nuclei and cytoplasm. Patchy p16 positivity can be seen in the endometrial stroma (right upper corner). C In the same microscopic field as that of image $\mathbf{B}$, immunostaining for estrogen receptor highlighted the endometrial stromal cells, but not AIS. D The uninvolved endometrial glands and stroma reacted uniformly with anti-progesterone receptor antibody. Staining method: A-D, polymer method (immunostaining). Magnification: A, $\times 40$; B-D, $\times 200$.

(Fig. 2G-H), whereas the adjacent endometrial glandular epithelium showed patchy p16 positivity (Fig. 2I). The final pathological diagnosis was superficially spreading endocervical AIS with microscopic multifocal involvement of the endometrial surface epithelium.

All available previous slides from the endometrial curettage specimen were reviewed. The foci of atypical glandular proliferation, initially misinterpreted as EEC, were morphologically identical to those of endocervical AIS. A few areas had mitotic figures and apoptotic bodies, although they were not readily identifiable. Immunostaining revealed that the atypical glands exhibited block p16 positivity, confirming the involvement of endocervical AIS (Fig. 3A-B). In contrast, immunostaining for estrogen receptor (ER) and progesterone receptor (PR) highlighted the endometrial glands and stroma only (Fig. 3C-D).

The patient did not receive further treatment. At the first postoperative outpatient visit, she was well without any evidence of disease recurrence. She was referred to another tertiary hospital to continue postoperative care closer to home.

\section{Discussion}

It is essential to distinguish the endometrial involvement in EAC versus EEC for appropriate pathological staging and clinical management. However, it is difficult to differentiate between the endometrial versus endocervical origin of small tumor fragments from the 
curettage specimens by their morphological features alone. The distinction between EAC and EEC is still challenging due to the following [7]: First, both tumors have mucinous and endometrioid-like features. The EAC is most often characterized by a hybrid of mucinous and endometrioid-like features, usually with increased mitotic figures and apoptosis. Some of the EECs can also have varying degrees of mucinous differentiation. Second, both tumors most often exhibit entirely well-differentiated glandular proliferation and a villoglandular growth pattern. Third, when tumors involve both the endometrium and the endocervix, the interpretation of small tissue fragments from biopsy or curettage specimens is difficult. For example, an AIS or EAC extending into the endometrium simulates atypical hyperplasia/endometrioid intraepithelial neoplasia or endometrioid carcinoma, and EEC involving the endocervix closely resembles EAC. The diagnosis of superficially spreading AIS with multifocal microscopic endometrial involvement requires careful histological examination of the uterine body and cervix to exclude the possibility of EEC.

Nevertheless, the following histological features help distinguish EAC from EEC: a higher degree of nuclear atypia, markedly increased mitotic activity, frequent apoptotic bodies, and the absence of squamous morules [7,8]. Compared with EEC, the EAC is more likely to exhibit a higher degree of nuclear atypia, including moderate-to-severe pleomorphism and hyperchromasia, often with numerous apically situated mitotic figures and basally situated apoptotic bodies. Atypical mitotic figures are also frequently seen in EAC but are very rare in lowgrade EEC. The foci of squamous differentiation (squamous morules) are more frequently found in EEC but absent in EAC.

In routine practice, immunostaining with a few selected markers can also readily distinguish between EECs and EACs. The most useful marker is p16, followed by ER and PR [1]. p16 immunoreactivity is a surrogate marker for high-risk HPV infection [9]. HPV-associated EAC almost always shows block positivity for $\mathrm{p} 16$, compared to the variable and non-diffuse positivity seen in low-grade EEC. While most EECs express ER and PR, most EACs exhibit focal and weak ER expression and a loss of PR expression.

In summary, EAC can be misdiagnosed as EEC when evaluating small tumor tissues in the endometrial curettage, particularly when the pathologists are unaware of the possibility of microscopic endometrial involvement of EAC. In most cases of a suspected endometrial lesion, their origin can be correctly determined based on a combination of histological features and immunostaining results, even when the former is based upon the examination of relatively small samples. The presence of frequent mitotic figures and apoptotic bodies indicate endocervical AIS since endometrial low-grade endometrioid carcinoma generally do not have the notable mitotic activity and apoptotic bodies. Accurate diagnosis in these cases is very important because it has significant implications on pathological staging, management, and prognosis.

\section{Acknowledgment}

We would like to thank Dr. Sung-Im Do (Department of Pathology, Kangbuk Samsung Hospital, Sungkyunkwan University School of Medicine, Seoul, Republic of Korea) for her valuable comments and support.

\section{Statement of Ethics}

Written informed consent for publication was obtained from the patient. This study was reviewed and approved by the Institutional Review Board of Samsung Medical Center (Seoul, Republic of Korea) (2020-07-116).

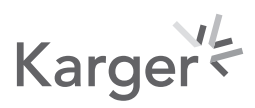




\section{Conflict of Interest Statement}

The authors have no conflicts of interest to declare.

\section{Funding Sources}

This research was supported by the National Research Foundation of Korea (NRF) grant funded by the Korean Government (Ministry of Science and ICT) (2018R1C1B5043725).

\section{Author Contributions}

Inwoo Hwang: conceptualization, data collection, data analysis, and manuscript drafting; Jiyeon Lee: data collection and manuscript editing; Kyue Hee Choi: data analysis and manuscript editing; Jiheun Han: manuscript editing; Hyun-Soo Kim: conceptualization, data analysis, manuscript drafting, manuscript editing, funding acquisition, and supervision. All authors read and approved the final manuscript.

\section{References}

1 Buza N, Hui P. Immunohistochemistry in Gynecologic Pathology: An Example-Based Practical Update. Arch Pathol Lab Med. 2017 Aug;141(8):1052-71.

2 Kim HS, Chung YS, Kim MS, Ryu HJ, Lee JH. Endocervical Adenocarcinoma In Situ Phenotype with Ovarian Metastasis. J Pathol Transl Med. 2019 Jul;53(4):270-2.

3 Chang MC, Nevadunsky NS, Viswanathan AN, Crum CP, Feltmate CM. Endocervical adenocarcinoma in situ with ovarian metastases: a unique variant with potential for long-term survival. Int J Gynecol Pathol. 2010 Jan; 29(1):88-92.

4 Reyes C, Murali R, Park KJ. Secondary Involvement of the Adnexa and Uterine Corpus by Carcinomas of the Uterine Cervix: A Detailed Morphologic Description. Int J Gynecol Pathol. 2015 Nov;34(6):551-63.

5 Khor TS, Brennan BA, Leung YC, Sengupta S, Stewart CJ. Cervical adenocarcinoma metastatic to the ovary mimicking primary ovarian carcinoma. Pathology. 2009;41(4):397-400.

6 Turashvili G, Farmer P, Colgan T, Childs T. Human Papillomavirus-Related Ovarian Metastasis With Endocervical Adenocarcinoma: Report of 2 Cases and Review of Literature. J Low Genit Tract Dis. 2015 Oct;19(4): e60-3.

7 Ronnett BM. Endocervical adenocarcinoma: selected diagnostic challenges. Mod Pathol. 2016 Jan;29(Suppl 1):S12-28.

8 Stewart CJR, Crum CP, McCluggage WG, Park KJ, Rutgers JK, Oliva E, et al. Guidelines to Aid in the Distinction of Endometrial and Endocervical Carcinomas, and the Distinction of Independent Primary Carcinomas of the Endometrium and Adnexa From Metastatic Spread Between These and Other Sites. Int J Gynecol Pathol. 2019 Jan;38(Suppl 1):S75-92.

9 Keating JT, Ince T, Crum CP. Surrogate biomarkers of HPV infection in cervical neoplasia screening and diagnosis. Adv Anat Pathol. 2001 Mar;8(2):83-92.

\section{Karger'"}

